\title{
Le modèle républicain d'intégration : implications pour la psychologie des relations entre groupes
}

\author{
Rodolphe Kamiejski*, Serge Guimond*, Pierre De Oliveira, \\ Abdelatif Er-Rafiy et Markus Brauer \\ Clermont Université, Université Blaise Pascal, Laboratoire de Psychologie Sociale et \\ Cognitive (UMR 6024 CNRS)
}

\section{RÉSUMÉ}

Des analyses historiques et sociologiques ont mis en évidence les caractéristiques du modèle républicain en tant que tradition spécifiquement française d'intégration et d'organisation de la vie collective. Cependant, très peu de recherches en psychologie ont examiné les perceptions, les attitudes ou les croyances des individus à l'égard de ce modèle. Nous présentons les résultats d'une série d'études qui mettent en évidence deux dimensions distinctes et orthogonales structurant l'idéologie républicaine, l'une reliée à la citoyenneté et l'autre reliée à la laïcité. De plus, nous montrons que l'adhésion à ces principes républicains est non seulement très élevée mais qu'elle a des implications importantes pour comprendre les perceptions et les attitudes intergroupes des Français(es), ainsi que les orientations d'acculturation de Français(es) issus de l'immigration maghrébine.

The republican model of integration: Implications for the psychology of intergroup relations

\section{ABSTRACT}

Historical and sociological analyses have highlighted the characteristics of the republican model as a specifically French tradition of integration and organization of collective life. However, few research in psychology has examined the perceptions, attitudes or beliefs of individuals with regard to this model. We present the results of several studies that reveal two distinct and orthogonal dimensions structuring the Republican ideology : one related to citizenship and the other related to secularism. Moreover, we show that

*Correspondance : Rodolphe Kamiejski ou Serge Guimond, UFR de Psychologie, LAPSCO CNRS 6024, 34 avenue Carnot, 63037 Clermont-Ferrand. Email : Rodolphe.Kamiejski@etudiant.univ-bpclermont.fr ; serge.guimond@univ-bpclermont.fr

Remerciements. Cette recherche a été rendue possible grâce à un octroi de l'Agence Nationale de la Recherche (ANR-06-CONF-07). 
adherence to Republican principles is not only very high but has important implications for understanding the intergroup perceptions and attitudes of French people, and the acculturation orientations of French immigrants from North Africa.

«Dans l'histoire de France, le respect de l'égalité entre territoires sous l'Ancien Régime et entre les citoyens devant la loi depuis la Révolution est au cour de la construction de la nation. L'égalité reste à la fois un principe et un programme au cœur de l'identification des Français à leur État et à leur nation » (Weil, 2008, p. 22).

À l'heure où ressurgissent dans le débat politique les questions d'identité nationale, de statistiques ethniques ou encore d'affirmation de principes républicains, il paraît opportun de s'interroger sur un modèle Républicain français érigé depuis des décennies en exemple d'intégration. Modèle en crise pour certains (Streiff-Fénart, 2002), illustré au travers des émeutes dites de banlieues fin 2005 (Furedi, 2005), il n'en reste pas moins qu'à notre connaissance aucun travail scientifique de psychologie sociale n'a jusqu'à ce jour cherché à mesurer de manière systématique ce qu'un tel système déterminait réellement. On peut notamment s'interroger à savoir si ce modèle qui se veut garant de la liberté, de l'égalité et de la fraternité favorise efficacement acceptation, respect et intégration pour ses citoyens et en particulier pour ses minorités.

En effet, contrairement à une documentation fournie concernant le modèle républicain en sociologie, en histoire ou en sciences politiques (par exemple, Mayer, Michelat, \& Tiberj, 2008 ; Schnapper, 2007 ; Weil, 2007), on peine à trouver une seule recherche empirique publiée sur ce thème en psychologie. Pourtant, pour comprendre la psychologie des relations entre groupes en France, il est probablement essentiel de considérer comment les individus se positionnent face au modèle républicain. Dans ce contexte, une série de recherches a été réalisée avec quatre principaux objectifs : 1. développer un instrument de mesure de l'adhésion des individus aux principes du modèle républicain d'intégration ; 2 . vérifier les relations entre l'adhésion à ce modèle et les attitudes envers le multiculturalisme, un modèle généralement considéré comme l'antithèse du modèle Républicain ; 3. déterminer dans quelle mesure l'adhésion à ces modèles d'intégration favorise l'ouverture et la tolérance à l'égard d'autrui ou, au contraire, le racisme et la xénophobie ; et enfin 4 . à partir de la perspective de Français issus de l'immigration maghrébine, amorcer l'étude des relations entre l'adhésion aux principes du modèle républicain et les stratégies d'acculturation. 


\section{LE MODÈLE RÉPUBLICAIN : L'INTÉGRATION À LA FRANÇAISE}

Le modèle républicain français repose sur un système politique où l'identité collective est créé par une adhésion volontaire de la communauté des citoyens à des principes et valeurs partagées (Bénichou, 2006). La France affiche ainsi les termes de son contrat pour la citoyenneté dans l'article premier de la Constitution française de 1958 déclarant que : «La France est une République indivisible, laïque, démocratique et sociale ». En s'appuyant sur ces quatre fondements, ce système vise à assurer l'union fraternelle d'une nation de citoyens libres et égaux en droits. Mais ce projet politique fixe également par sa définition certaines limites aux droits et reconnaissances qu'il accorde. Ainsi, il affirme que le citoyen est l'unité de base de la République, avec pour conséquence, que seuls les individus et non les communautés soient reconnus et intégrés (principe d'indivisibilité). Autre principe fondateur, la laïcité s'accorde avec l'indivisibilité de la nation sur la place fondatrice (voire exclusive) de l'individu, en incitant au dépassement des particularismes originels et notamment religieux. Ces deux principes affirment que « le lien social doit transcender les liens sociaux primaires » (Reynier, 2000). Seulement, ces principes, piliers du modèle français, se confrontent à une réalité souvent complexe (économique, migratoire et sociale), et bien différente de celle qui prévalait lors de leur édiction en 1958 (voir Bertossi, 2006 pour une perspective historique du modèle républicain). Ce système révèle dans les faits un certain nombre de dysfonctionnements au regard de ce qu'il promettait initialement.

Ainsi, l'intégration égalitaire prônée se trouve fortement mise à mal par l'existence de discriminations multiples (logement, travail, loisirs ...) rencontrées par les franges les plus fragiles de la population (immigrés, chômeurs ... : Beauchemin, Hamel, Lesné, Simon, et al., 2010). Les études réalisées par Amadieu (2004, 2006) pour l'Observatoire des Discriminations viennent rendre compte à ce titre d'un tel phénomène. Utilisant la méthode du testing, il montre par exemple qu'un candidat dit «de référence » (homme, patronyme français, blanc, sans traits particulièrement disgracieux) aura entre trois et cinq fois plus de chance d'être convoqué à un entretien d'embauche qu'un candidat au patronyme maghrébin. Les Nord-Africains sont même une cible privilégiée d'hostilité en France (Pettigrew, Jackson, Ben Brika, Lemaine, Meertens, Wagner, \& Zick, 1998). Le modèle républicain qui montre des difficultés, dans sa mise en œuvre, à prémunir ses citoyens face à ces injustices, porte également une certaine responsabilité dans le malaise ambiant au travers de ses orientations à l'efficacité discutable. Illustrations concrètes de telles 
situations, citons par exemple l'interdiction de produire en France des statistiques d'ordre ethnique (loi du 6 janvier 1978) ou bien encore l'intransigeance de l'État français face au port du voile à l'école (loi du 15 mars 2004). Ces mesures se voient contestées car elles seraient en fait susceptibles de masquer l'ampleur de certaines réalités (inégalités, exclusions. ..) vécues par ceux que la République s'est donnée pour mission de protéger. Comme le précise Reynier (2000), « initialement, la laïcité n'est pas pensée contre la religion » mais, comme le rapporte l'Observatoire Européen des Phénomènes Racistes et Xénophobes (EUMC) en 2006 sur la perception de la discrimination et de l'islamophobie par les membres des communautés musulmanes : « en France, les personnes interrogées estiment que l'interdiction dans les écoles publiques [du voile] a contribué à la création d'un climat d'hostilité envers les musulmans ». On est donc en droit de se demander si dans une plus large mesure, le principe de laïcité ne se traduit pas dans les faits par une certaine intolérance envers les religions et en particulier aujourd'hui à l'égard de l'Islam (voir Barthélemy \& Michelat, 2007 ; Brouard \& Tiberj, 2005 ; Mayer \& Michelat, 2007). Ainsi sous un angle plus politique, alors qu'historiquement ce principe a pu constituer un élément fondamental de l'identité de gauche, depuis 1989 l'absence de vision commune de la laïcité n'en structure plus l'identité. La « défense de la laïcité menacée » est désormais reprise par une partie de la droite, des laïcs de droite étant plus intolérants que ceux de gauche (Baubérot, 2010 ; Barthélémy \& Michelat, 2007).

$\mathrm{Au} \mathrm{vu}$ des diverses contradictions soulevées au sujet du modèle républicain français, mais aussi de l'ambiguïté liée à son interprétation, il semble assez indiqué de chercher à comprendre plus exactement ce qu'il recouvre. Cela implique notamment de définir précisément ses contours, son contenu. Et également, de chercher à savoir si ce modèle fondé sur la place de l'individu préserve efficacement des haines et des conflits, et possède une influence positive réelle sur les relations intergroupes, à l'instar d'autres modèles influents tels que le multiculturalisme. Or, si les effets spécifiques liés au modèle républicain sont peu connus, de nombreux travaux issus de la psychologie sociale, plaçant de manière centrale la question des relations intergroupes et de leur nature conflictuelle nous permettent d'entrevoir certains de ses aspects.

\section{LES MODĖLES DU CONTACT INTERGROUPE EN PSYCHOLOGIE SOCIALE}

Des chercheurs pionniers de la discipline tels qu'Allport (1954) ou bien encore Sherif et Sherif (1953) se sont intéressés dès les années 
1950 à la façon de limiter les réactions de rejet, de discrimination et d'exclusion observées entre membres de différents groupes. En examinant les principales théories de psychologie sociale concernant ces processus, on peut établir certaines correspondances entre ces théories et certaines politiques d'intégration très contrastées telles que l'approche républicaine ou le modèle du multiculturalisme.

Le modèle de la personnalisation. Dans une perspective devenue classique, Brewer et Miller (1984) ont proposé le modèle de la personnalisation qui fixe les conditions sous lesquelles le contact entre les membres de groupes distincts devrait diminuer les préjugés et favoriser l'acceptation mutuelle. Puisque la catégorisation "nous/eux » est à la base de la discrimination et des préjugés (voir Tajfel, 1970 ; Tajfel \& Turner, 1979), Brewer et Miller (1984) suggèrent que pour réduire cette tendance discriminatoire, il faut éviter le plus possible de recourir à la catégorisation sociale. La méthode proposée se nomme donc décatégorisation-personnalisation (Brewer \& Miller, 1984) et recherche l'élimination psychologique des catégories existantes (Park \& Judd, 2005). Elle préconise qu'une personne en situation de contact doit s'attacher à ne percevoir autrui que par le biais d'éléments personnalisants, l'amenant alors progressivement à ne le traiter qu'en individu unique, et non plus en tant que représentant d'un groupe. Ceci devrait donc conduire à réduire la saillance catégorielle originelle de la cible, et à la suite d'interactions coopératives, à générer des attitudes plus favorables (voir Miller, 2002).

La perception purement individualisante de cette méthode rappelle donc de manière frappante ce que l'on énonçait précédemment au sujet du modèle républicain français : pour préserver l'absence de distinction entre ses citoyens (Guimond, 2004) et l'entente sociale, il ne reconnait pas l'existence de sous-parties de la population et positionne l'individu comme pivot du système. Comme le rappelle Schnapper (2000) « l'intégration sociale s'est faite en France autour de l'idée de citoyenneté individuelle ».

Selon le modèle de Brewer et Miller (1984), on pourrait donc s'attendre à ce que les individus qui, en France, adhèrent le plus au principe de décatégorisation qui caractériserait le modèle républicain, soient aussi celles et ceux qui manifestent le moins de préjugés intergroupes. Un des objectifs des recherches présentées ici sera précisément d'examiner cette proposition.

"Le modèle de la différentiation intergroupe mutuelle ». Un certain nombre de critiques ont été adressées au modèle de Brewer et Miller (1984). En fait, on peut se demander si ce modèle ne va pas à l'encontre d'une bonne partie des connaissances scientifiques en psychologie au sujet du processus de catégorisation. En effet, la catégorisation fait partie intégrante de notre fonctionnement cognitif. Il ne semble pas possible d'en 
faire l'économie, comme le voudrait le principe de décatégorisation. Au contraire, toute perception impliquerait, selon Bruner (1957), un processus de catégorisation. De plus, il est maintenant bien établi que la catégorisation d'autrui, en fonction d'indices visuels, auditifs ou comportementaux, est un processus extrêmement rapide et quasiment automatique (Brauer, Wasel, \& Niedenthal, 2000 ; Devine, 1989 ; Schadron \& Morchain, 2008). Enfin, les travaux reliés à «l'effet rebond » montrent que les efforts cognitifs visant à ne pas catégoriser et à ne pas utiliser ses stéréotypes, comme le suggère le modèle de la personnalisation, ont souvent l'effet exactement inverse : nos jugements et nos comportements se trouvent encore plus influencés par la catégorisation sociale dans de telles conditions (voir Macrae, Bodenhausen, Milne, \& Jetten, 1994).

Face à ces problèmes, Hewstone et Brown (1986) ont proposé un modèle alternatif connu sous le nom de modèle de la différentiation intergroupe mutuelle. Ce modèle propose une méthode prenant le contre-pied de la logique précédente puisqu'elle postule que la conservation et la reconnaissance des appartenances catégorielles résoudraient la relation conflictuelle à autrui. Autrement dit, ce modèle suggère, à l'inverse du modèle de Brewer et Miller (1984), qu'étant donné les besoins psychologiques importants auxquels répond l'appartenance à un groupe, il serait contre-productif de nier cette identité. Et pour aboutir à des relations positives entre groupes, cela nécessiterait de catégoriser et reconnaître les identités de chacun.

Ce que préconise le modèle de la différentiation mutuelle se retrouve, au niveau des politiques d'intégration, dans l'approche du multiculturalisme adoptée notamment au Canada (Guimond, 1999). Selon cette approche, l'État se doit de prendre en compte et de valoriser les multiples identités des communautés culturelles composant son espace. Les fonds publics sont donc utilisés pour faire la promotion de la diversité culturelle au sein de la nation. À partir de la fin des années 1970, les psychologues sociaux canadiens ont effectué de nombreuses recherches sur les attitudes envers le multiculturalisme (voir Berry \& Kalin, 1995 ; Berry, Kalin, \& Taylor, 1977). Plus récemment, des recherches analogues ont été conduites principalement aux États-Unis (Wolsko, Park, \& Judd, 2006) et aux Pays-Bas (Verkuyten, 2006). Les résultats montrent très clairement que l'adhésion au multiculturalisme est reliée à une diminution des préjugés intergroupes - ce constat a même été confirmé de manière expérimentale (voir Richeson \& Nausbaum, 2004 ; Verkuyten, 2005).

Assez peu de recherches ont été menées sur les attitudes envers le multiculturalisme (MC) en France (voir Lambert, Moghaddam, Sorin, \& Sorin, 1990 ; Maisonneuve \& Testé, 2007 ; Sabatier \& Berry, 1999). Pourtant, 
la situation française offre un grand intérêt à cet égard. En effet, il serait important de savoir si, dans un pays sous l'influence d'un modèle opposé au multiculturalisme (voir Sala Pala \& Simon, 2007), on retrouve des résultats similaires, à savoir une relation positive entre l'adhésion au MC et une plus grande acceptation des autres groupes. Contrairement à ce que l'on pourrait penser, Lambert et collaborateurs (1990) n'ont pas observé un rejet global du MC et un appui massif pour l'assimilation en France. De plus, ils ont montré que l'orientation politique était déterminante, les personnes de gauche ayant des attitudes beaucoup plus favorables à l'égard du MC que les personnes de droite. La question du rôle de l'orientation politique sera donc également examinée dans la présente recherche.

\section{MODÈLES POLITIQUES D'INTÉGRATION ET ACCULTURATION : PERSPECTIVE DES MINORITÉS CULTURELLES}

La compréhension des processus d'intégration et de la mesure dans laquelle un modèle d'intégration particulier favorise effectivement des relations harmonieuses entre les groupes réclame de prendre en considération non seulement le point de vue des groupes majoritaires ou dominants mais également celui des minorités culturelles (Berry, 2005). La politique du MC par exemple qui vise la promotion de la diversité culturelle n'a de sens que si les gens d'origine diverse souhaitent effectivement maintenir vivantes leurs caractéristiques culturelles d'origine. Comment les personnes d'origine maghrébine, en France, réagissent-elles face au modèle républicain et au multiculturalisme ? Le modèle des stratégies d'acculturation de Berry $(1994 ; 2005)$ permet de conceptualiser cette problématique et de clarifier un certain nombre d'enjeux importants pour comprendre le débat public en France. Selon ce modèle, les membres d'un groupe minoritaire doivent répondre à deux questions fondamentales : a) Y a-t-il lieu de valoriser l'identité et les caractéristiques culturelles d'origine ? et b) Y a-t-il lieu de valoriser les relations avec les autres groupes dans la société ? Les réponses à ces deux questions peuvent être complexes mais à des fins conceptuelles, elles sont traitées dans le modèle comme des préférences dichotomiques (« oui » ou « non » à chaque question), générant ainsi quatre principales stratégies d'acculturation : l'intégration (oui/oui), l'assimilation (non/oui), la séparation (oui/non) et la marginalisation (non/non).

Une première utilité de ce modèle est de montrer que, contrairement au discours médiatique ambiant qui fait référence au terme d'intégration 
en sous-entendant bien souvent l'assimilation, il est possible de distinguer ces deux options (voir Sabatier \& Berry, 1999). L'assimilation partage avec l'intégration la volonté d'appartenir à la communauté nationale, mais s'en distingue par le fait de ne pas souhaiter conserver les caractéristiques culturelles d'origine. De ce fait, l'intégration correspond à l'option du multiculturalisme. Ainsi, les personnes qui adoptent une orientation d'intégration ont habituellement des attitudes positives envers le multiculturalisme (Berry, 2005 ; Guimond, 2010).

Deuxième utilité du modèle, il permet de dissiper la confusion entretenue dans certains milieux en France par l'équation trompeuse multiculturalisme $=$ communautarisme. Le communautarisme fait référence à un groupe qui vit replié sur lui-même, en communauté. La plus grande enquête réalisée en France pour étudier le communautarisme, celle de Brouard et Tiberj (2005), a montré que «le communautarisme au sens strict, quel que soit l'angle par lequel on l'étudie, ne concerne qu'une frange extrêmement minoritaire des Français issus d'Afrique et de Turquie » (p. 134). À la lumière de la façon dont Brouard et Tiberj (2005) ont procédé pour étudier la question, il apparaît que le communautarisme correspond à l'orientation de séparation, et non à celle d'intégration (qui correspond au multiculturalisme). En d'autres termes, quand on parle en France du danger du communautarisme, on parle essentiellement des problèmes occasionnés par des gens qui adoptent cette orientation de séparation, refusant tout mouvement vers la population nationale et insistant pour maintenir un contact quasi exclusif avec leur groupe d'origine (voir Guimond, 2010). Cette orientation de séparation ne correspond évidemment pas à celle du multiculturalisme. Cette proposition est importante car elle signifie, étant donné qu'il existe déjà des instruments pour mesurer cette orientation de séparation, qu'on pourrait étudier le communautarisme en France à l'aide de ces mêmes mesures. Enfin, à quoi peut donc correspondre dans le modèle de Berry une politique comme celle du modèle républicain ? La plupart des auteurs suggèrent que le modèle républicain est essentiellement un modèle assimilationniste et qu'il correspond, à ce titre, à l'orientation d'assimilation du modèle de Berry (Sabatier \& Berry, 1999). En fait, aucune étude systématique de cette question n'a encore été réalisée à notre connaissance. Par conséquent, un objectif important de notre troisième étude était d'examiner les relations entre le degré d'adhésion aux principes du modèle républicain et les orientations d'acculturation des Français issus de l'immigration maghrébine.

L'autre question à laquelle nous nous intéresserons est celle de l'effet de l'appartenance ethnique de l'expérimentateur. Au travers de l'analyse 
des travaux réalisés dans le domaine des stratégies d'acculturation (par ex., Barrette, Bourhis, Moïse, Personnaz, \& Personnaz, 2004 ; Verkuyten \& Kwa, 1996), on peut constater que les participants issus des minorités ethniques sont souvent questionnés par un expérimentateur appartenant au groupe majoritaire. Il reste alors possible que les participants de minorités ethniques fournissent des réponses conformes aux attentes de la population d'accueil dont est issu l'expérimentateur. Par conséquent, et en accord avec certains principes méthodologiques de base (Guimond, 2010), l'origine ethnique de l'expérimentateur a été rigoureusement contrôlée.

En résumé, une série de recherches a été conduite afin d'examiner dans quelle mesure les principes du modèle républicains d'intégration peuvent se traduire dans des attitudes et prises de position de nature à éclairer les rapports entre groupes majoritaires et minorités culturelles en France. Pour ce faire, notre point de départ a été de développer un instrument permettant de mesurer l'adhésion des individus aux principes du modèle républicain.

\section{PRÉ-TEST}

Comme le rappelle Felouzis (2006) : «La République française ne reconnaît dans ses principes actuels que des citoyens « libres et égaux» en dehors de toutes considérations d'origine culturelle, de religion ou d'appartenance communautaire » (p. 318). En consultant des sources diverses et variées (ouvrages académiques, journaux, sites internet), nous avons développé un questionnaire contenant une série de 25 énoncés affirmant des positions caractéristiques de cette idéologie républicaine (c'est-à-dire « Je ne veux pas qu'on définisse les gens en France en fonction de leurs origines, de leur religion. »). Dans le cadre d'une pré-enquête, ce questionnaire a été soumis à 70 étudiants en psychologie, dont $78 \%$ de femmes, qui devaient indiquer leur degré d'accord avec les différents énoncés sur une échelle de 1 (pas du tout d'accord) à 7 (tout à fait d'accord). Ils devaient de la même façon indiquer leur orientation politique gauche/droite (voir Étude 1 pour la mesure dont le score élevé signifiera un positionnement à gauche, $M=4,91, E T=1,64)$.

Une analyse en composantes principales a été réalisée sur les items destinés à mesurer l'adhésion aux principes républicains. De cette analyse ressortent deux facteurs indépendants expliquant plus de $50 \%$ de la variance. Les énoncés ayant une forte saturation sur le facteur 1 nous ont conduit à nommer ce facteur « Citoyenneté » (ex. « La société française 
est composée de citoyens avant toute chose et non pas de communautés »). Une échelle construite à partir de ces items présente une consistance interne satisfaisante $(\alpha=0,75)$. Les énoncés ayant une forte saturation sur le facteur 2 nous ont conduits à nommer ce facteur «Laïcité » (ex. " Il me semble tout à fait normal que dans les écoles publiques en France les signes religieux visibles soient interdits »). Une échelle construite à partir de ces items présente aussi une consistance interne satisfaisante $(\alpha=0,67)$.

L'analyse des corrélations entre citoyenneté et laïcité révèle que les deux variables ne sont pas corrélées significativement $(r=-0,17, n s)$, suggérant que nous sommes donc en présence de deux dimensions orthogonales. De plus, ces deux mesures de l'adhésion aux principes du modèle républicain n'ont aucun rapport avec l'orientation politique. En effet, que les participants se disent de droite ou de gauche, les corrélations entre l'orientation politique et les échelles de citoyenneté ou de laïcité ne sont jamais statistiquement significatives $(r s<0,17, n s)$. Trois études ont été menées afin de vérifier ces résultats préliminaires et de déterminer dans quelle mesure l'adhésion à ces principes républicains a des implications pour la compréhension des attitudes intergroupes et des préjugés. Les Études 1 et 2 s'intéresseront au point de vue de participants issus du groupe majoritaire en France, aux relations entre le multiculturalisme et l'adhésion aux principes du modèle républicain, ainsi qu'aux effets de cette adhésion pour les relations intergroupes. L'Étude 3 s'intéressera à la perspective des Français issus de l'immigration maghrébine.

\section{ÉTUDE 1}

Les items identifiés lors du pré-test, définissant deux dimensions de l'idéologie républicaine, ont été utilisés dans une première étude afin de vérifier si, effectivement, l'adhésion au modèle républicain est en contradiction avec une adhésion au MC. De plus, il s'agissait d'examiner les relations entre l'adhésion à ces modèles d'intégration et certaines attitudes intergroupes.

\section{Méthode \\ Participants}

L'enquête a été réalisée auprès de cent vingt-trois étudiants de première année de psychologie (dont $95 \%$ de femmes). Ces participants dont l'âge variait entre 17 et 
27 ans $(M=19$ ans, $E T=1,38)$ ont été interrogés à partir d'un questionnaire dans des conditions variées par un expérimentateur homme ou femme.

\section{Mesures}

Le questionnaire comprenait une série d'énoncés à propos desquels les participants devaient indiquer leur degré d'accord sur une échelle en 7 points $(1=$ « Pas du tout d'accord » et $7=$ « Tout à fait d'accord »). Ces différents énoncés incluaient les mesures suivantes :

- une échelle d'Orientation à la Dominance Sociale (ODS) de 8 items de Sidanius et Pratto (1999) validée en français par Duarte, Dambrun et Guimond (2004) : (« Améliorons l'égalité sociale » [score inversé] ; «Certains groupes sont tout simplement inférieurs aux autres groupes ») $\alpha=0,78$. Comme proposé par Jost et Thomson (2000) et confirmé en France par Duarte et al. (2004), cette échelle se décompose en deux facteurs. Un premier mesure l'opposition à l'égalité, (OEG) $(\alpha=0,78)$, et regroupe les items relatifs à l'égalité sociale. Le second concerne la dominance entre les groupes (DBG) $(\alpha=0,63)$, et regroupe tous les items valorisant la hiérarchie sociale ;

- une échelle de préjugés anti-Maghrébins de 9 items de Dambrun et Guimond (2001) (« On peut facilement comprendre la colère que ressentent les Maghrébins en France » [score inversé] ; « S'il y a beaucoup de chômage en France, c'est parce que les étrangers prennent le travail des Français ».) $\alpha=0,84$;

- une échelle d'attitude envers le multiculturalisme de 8 items de Berry et Kalin (1995) («Les gens qui viennent vivre en France devraient adapter leur comportement pour ressembler plus aux Français » [score inversé] ; «La vie serait plus agréable en France si les différents groupes ethniques y conservaient leur mode de vie bien vivant. ») $\alpha=0,78$;

- une mesure d'orientation politique : deux propositions demandaient aux participants d'indiquer leur degré d'accord-désaccord avec le fait de se considérer comme une personne de gauche et de droite. Le lien étroit et négatif entre ces deux énoncés $(r=-0,615)$, qui renvoie à la polarisation droite-gauche du contexte politique français, nous a conduits à les regrouper, après recodage de l'item droite, au sein d'une même mesure (un score élevé indiquera leur orientation politique à gauche);

- une série de 8 items sélectionnés à partir des résultats de la pré-enquête et destinés à mesurer l'adhésion au modèle républicain.

Les résultats d'une analyse en composantes principales sur les items républicains ont confirmé une structure en deux facteurs indépendants : un facteur « citoyenneté » se définissant par l'idée d'une banalisation des appartenances d'origine (ethnie, « race », religion, etc.) au profit d'une égalité citoyenne, et un facteur « laïcité » reflétant le désir de confiner à la sphère privée, et non publique, 
les manifestations d'appartenance religieuse. Deux échelles ont donc été construites à partir des items saturant le plus fortement sur chacun des deux facteurs. L'échelle de « citoyenneté » se compose de quatre items $(\alpha=0,61)$, l'échelle de laïcité $(\alpha$ $=0,53)$ également.

\section{Résultats}

\section{Adhésion aux modèles d'intégration}

La Figure 1 indique le niveau d'adhésion aux principes du modèle républicain et à ceux du multiculturalisme. Elle permet de constater que l'adhésion à la citoyenneté $(M=5,59)$ et à la laïcité $(M=5,39)$ est très élevée (moyenne sur une échelle de 1 à 7 ). Les principes du multiculturalisme sont également évalués de manière positive $(M=4,66)$, mais significativement moins que ceux du modèle républicain. Les t-tests à échantillons appariés comparant multiculturalisme et « citoyenneté » d'une part, $t(122)=12,03, p<0,001$, et multiculturalisme et « laïcité » d'autre part, $t(122)=5,01, p<0,001$, viennent en attester.

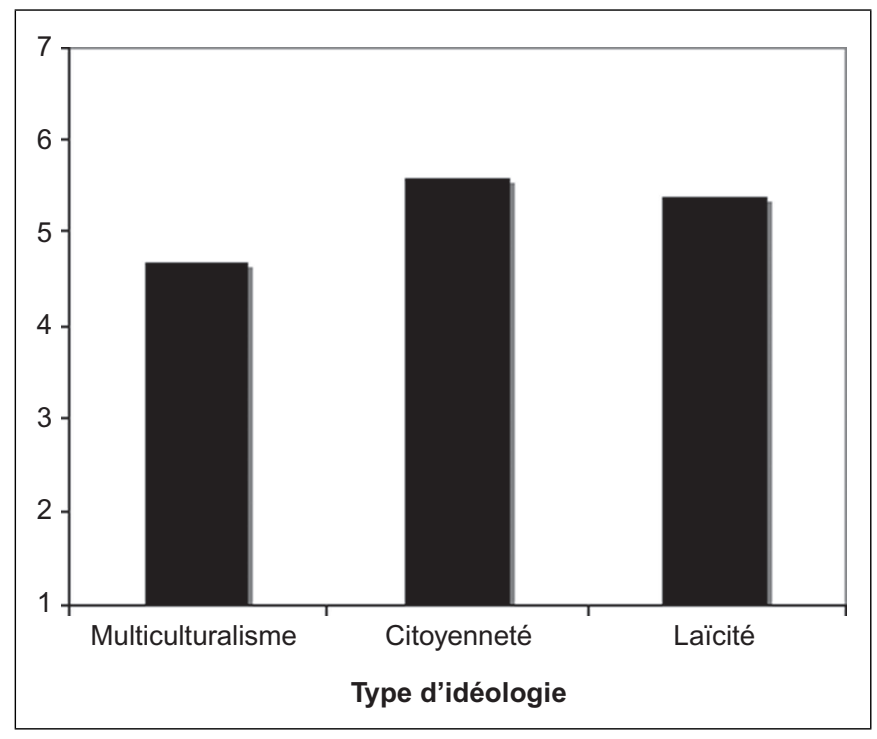

Figure 1. Niveau d'adhésion aux principes du modèle républicain et du multiculturalisme

Figure 1. Level of support for the principles of the republican model and multiculturalism 
Ces résultats sont en accord avec l'idée que la société française possède une tradition d'intégration spécifique, liée à son histoire nationale, et que nos mesures de citoyenneté et de laïcité en seraient des indicateurs appropriés.

\section{Liens entre les modèles d'intégration, les attitudes intergroupes et l'orientation politique}

Le Tableau 1 présente les corrélations obtenues entre les diverses mesures. On constate tout d'abord que les facteurs de citoyenneté et de laïcité sont indépendants et non-reliés, $r=0,01, n s$. De plus, ils sont corrélés différemment aux préjugés à l'égard des Maghrébins et à l'idéologie du multiculturalisme. L'adhésion au facteur « citoyenneté » est associée à de faibles préjugés, $r=-0,57, p<0,001$, tandis que l'adhésion au facteur "laïcité » renvoie de forts préjugés, $r=0,29, p<0,001$. Enfin, alors que le facteur « laïcité » est relié très négativement au multiculturalisme, $r=-0,39, p<0,001$, le facteur « citoyenneté » corrèle de manière positive à l'idéologie multiculturelle, $r=0,59, p<0,001$.

Tableau 1. Corrélations entre les facteurs du modèle républicain et diverses autres mesures

Table 1. Correlations between the factors of the republican model and various other measures

\begin{tabular}{|c|c|c|c|c|c|c|}
\hline & 1 & 2 & 3 & 4 & 5 & 6 \\
\hline $\begin{array}{l}\text { 1. Facteur } 1 \\
\text { Citoyenneté }\end{array}$ & - & & & & & \\
\hline 2. Facteur 2 Laïcité & 0,01 & - & & & & \\
\hline 3. Multiculturalisme & $0,59^{* * *}$ & $-0,39^{\star * *}$ & - & & & \\
\hline $\begin{array}{l}\text { 4. Préjugés } \\
\text { anti-Maghrébins }\end{array}$ & $-0,57^{\star * *}$ & $0,29^{\star * *}$ & $-0,71^{\star * *}$ & - & & \\
\hline 5. ODS & $-0,37^{\star * \star}$ & 0,12 & $-0,42^{\star * *}$ & $0,64^{\star * *}$ & - & - \\
\hline $\begin{array}{l}\text { 6. Orientation } \\
\text { politique }\end{array}$ & $0,42^{\star * *}$ & $-0,06$ & $0,50^{\star * \star}$ & $-55^{\star * *}$ & $-0,37^{\star * *}$ & - \\
\hline
\end{tabular}


De plus, ces deux mesures de l'adhésion aux principes du modèle républicain sont reliées de manière différente avec l'orientation politique des individus. En effet, plus ils se disent à gauche politiquement, plus ils sont citoyens et adhèrent au multiculturalisme. Par ailleurs, nous observons que moins ils se déclarent à gauche (plus ils se disent à droite), plus ils ont une haute ODS et de forts préjugés anti-Maghrébins.

On observe également que la laïcité ne possède de lien ni avec l'orientation politique, ni avec la dominance sociale $(r=0,12, n s)$. Il ne s'agirait donc pas d'une idéologie de légitimation. En revanche, la citoyenneté semble effectivement renvoyer à une dimension égalitaire puisqu'elle corrèle négativement avec la dominance sociale, $r=-0,37, p<0,001$. C'est ce que confirme le Tableau 2 qui distingue les deux composantes de l'ODS, la dominance basée sur les groupes et l'opposition à l'égalité (voir Duarte et al., 2004). Ce tableau apporte des précisions quant à la nature du lien entre ces variables et la citoyenneté grâce aux analyses de corrélations simples et partielles. Ces dernières ont pour objectif de vérifier dans quelle mesure l'adhésion au principe de citoyenneté renvoie à une question de dominance (facteur DBG de l'ODS), à une question d'égalité (facteur OEG de l'ODS), ou à ces deux questions conjointement. Les corrélations simples attestent donc d'une citoyenneté reliée de façon significative avec ces deux composantes, opposition à l'égalité, $r=-0,48, p<0,001$, et dominance basée sur les groupes $r=-0,18, p<0,05$. Cependant, les corrélations partielles montrent qu'en réalité, seul le lien négatif entre la citoyenneté et l'opposition à l'égalité se maintient lorsqu'on contrôle statistiquement l'autre composante de la dominance sociale, $r=-0,47, p<0,001$. Autrement dit, ces résultats montrent que l'échelle de citoyenneté a une orientation égalitaire qui n'a pas de rapport avec un désir de dominer les autres groupes.

Tableau 2. Corrélations simples et partielles entre facteur « citoyenneté » et les deux facteurs de l'ODS

Table 2. Simple and partial correlations between "citizenship" and the two SDO factors

\begin{tabular}{lcc}
\hline & ODS-égalité & ODS-dominance \\
\hline F1-Citoyenneté corrélation simple & $-0,48^{* * *}$ & $-0,18^{*}$ \\
\hline F1-Citoyenneté corrélation partielle & $-0,47^{* * *}$ & 0,09 \\
\hline $\begin{array}{l}\text { Note }: \\
{ }^{*} p<0,05 ;{ }^{* * *} p<0,001 .\end{array}$ & \\
\hline
\end{tabular}




\section{ÉTUDE 2}

Une seconde étude a été conduite afin de tester la généralité des résultats obtenus lors de l'Étude 1.

\section{Méthode \\ Participants}

Les résultats obtenus lors de l'Étude 1 et du pré-test pourraient être le reflet de caractéristiques propres aux étudiants en psychologie, majoritairement de sexe féminin (Henry, 2008 ; Paluck \& Green, 2009). Une seconde étude, semblable à la première, a donc été menée auprès d'un échantillon plus hétérogène et composé de cent quarante-cinq étudiants issus de diverses disciplines scientifiques (dont $48 \%$ de femmes). On y dénombre 69 femmes (âge moyen $=20,6$ ans, $E T=2,14$ ) et 74 hommes (âge moyen $=20,2$ ans, $E T=1,98$ ).

\section{Mesures}

Le questionnaire comprenait les mêmes mesures que pour l'Étude 1. Il s'agissait de

- l'échelle de citoyenneté composée de 4 items $(\alpha=0,78)$;

- l'échelle laïcité, avec 6 items $(\alpha=0,73)$;

- l'échelle multiculturalisme, 8 items $(\alpha=0,81)$;

- l'échelle de préjugés anti-Maghrébins, 9 items $(\alpha=0,83)$;

- l'échelle d'orientation à la dominance sociale (ODS), 8 items, $(\alpha=0,82)$;

- une mesure d'orientation politique (gauche/droite, $r=-0,75$ ).

\section{Résultats}

Dans l'ensemble, les résultats de cette deuxième étude confirment ceux précédemment obtenus. L'analyse en composantes principales des items républicains reproduit une structure en deux facteurs (voir Tableau 3 en annexe pour le détail de cette analyse).

\section{Adhésion aux modèles d'intégration}

La Figure 2 montre qu'auprès de cet échantillon également, composé d'hommes et de femmes, l'adhésion aux principes républicains est globalement très élevée, $(M=6,25$ pour la citoyenneté et $M=5,27$ pour la laïcité). Ici également, les t-tests à échantillons indépendants montrent que l'adhésion aux principes du multiculturalisme $(M=4.35)$ est significativement moins marquée que celle envers la citoyenneté, $t(122)$ 
$=20,84, p<0,001$, et la laïcité, $t(122)=5,82, p<0,001$. Cependant, on note un effet du genre sur l'adhésion à ces principes. L'effet s'avère significatif sur la laïcité, $F(1,143)=6,47, p<0,05$, où les hommes $(M=5,23)$ se montrent plus laïcs que les femmes $(M=5,05)$. Il n'est en revanche que tendanciel sur la citoyenneté $F(1,143)=2,97, p=0,087$ et sur le multiculturalisme $F(1,143)=3,43, p=0,066$ : les participantes adhèrent tendanciellement plus à la citoyenneté $(M=6,38)$ et au multiculturalisme $(M=4,48)$ que les participants $(M=6,14$ pour le premier, $M=4,17$ pour le second).

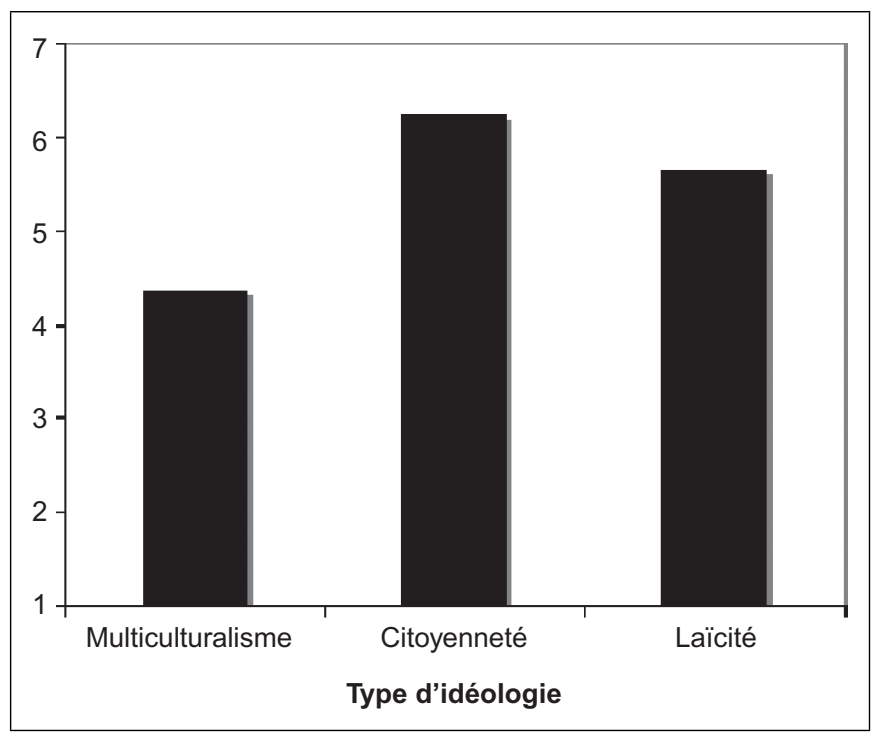

Figure 2. Niveau d'adhésion aux principes du modèle républicain et du multiculturalisme

Figure 2. Level of support for the principles of the republican model and multiculturalism

\section{Liens entre les modèles d'intégration et les attitudes intergroupes}

L'analyse des corrélations entre les variables confirme que les deux facteurs de l'idéologie républicaine sont indépendants et non-corrélés, $r=0,06$, ns (voir Tableau 4).

Comme pour l'Étude 1, l'adhésion au facteur « citoyenneté » renvoie à de faibles préjugés, $r=0,30, p<0,001$, alors que l'adhésion au facteur 
Tableau 4. Corrélations entre les facteurs du modèle républicain et les autres mesures

Table 4. Correlations between the factors of the republican model and other measures

\begin{tabular}{|c|c|c|c|c|c|c|}
\hline & 1 & 2 & 3 & 4 & 5 & 6 \\
\hline 1. Citoyenneté & - & & & & & \\
\hline 2. Laïcité & $-0,06$ & - & & & & \\
\hline 3. Multiculturalisme & $0,33^{* * *}$ & $-0,48^{\star \star \star}$ & - & & & \\
\hline $\begin{array}{l}\text { 4. Préjugés } \\
\text { anti-Maghrébins }\end{array}$ & $-0,30^{\star * *}$ & $0,46^{\star \star \star}$ & $-0,64^{\star * *}$ & - & & \\
\hline 5. ODS & $-0,42^{\star * *}$ & 0,13 & $-0,35^{\star * *}$ & $0,46^{* * *}$ & - & - \\
\hline $\begin{array}{l}\text { 6. Orientation } \\
\text { politique }\end{array}$ & $0,23^{* *}$ & $-0,17^{\star}$ & $0,40^{\star * *}$ & $-0,48^{\star \star \star}$ & $-0,43^{\star \star \star}$ & - \\
\hline
\end{tabular}

« laïcité » est associée à de plus forts préjugés, $r=0,46, p<0,001$. De plus, alors que le facteur "laïcité » est relié très négativement au multiculturalisme, $r=-0,48, p<0,001$, le facteur « citoyenneté » corrèle de manière positive avec l'idéologie multiculturelle, $r=0,33, p<0,001$. Enfin, on constate que la laïcité n'entretient aucun lien avec la dominance sociale, $r=0,13, n s$, alors que la citoyenneté est reliée à la composante de l'orientation à la dominance sociale qui traite de l'égalité et n'a pas de rapport avec un désir de dominer les autres groupes. Là encore, seul le lien négatif entre la citoyenneté et l'opposition à l'égalité se maintient lorsqu'on contrôle statistiquement l'autre composante de la dominance sociale, $r=-0,47, p<0,001$ (voir Tableau 5).

\section{Discussion}

Notre premier objectif devait permettre la création d'un outil mesurant l'adhésion aux principes du modèle républicain d'intégration. La cohérence des résultats obtenus dans les deux premières études indique qu'il est important de distinguer deux principes lui étant reliés : la citoyenneté républicaine, facteur distinct et indépendant du principe de laïcité. Le 
Tableau 5. Corrélations simples et partielles entre facteur « citoyenneté » et les deux facteurs de l'ODS

Table 5. Simple and partial correlations between "citizenship" and the two SDO factors

\begin{tabular}{lcc}
\hline & ODS-égalité & ODS-dominance \\
\hline F1-Citoyenneté corrélation simple & $-0,51^{\star * *}$ & $-0,24^{\star}$ \\
\hline F1-Citoyenneté corrélation partielle & $-0,47^{\star * *}$ & 0,08 \\
\hline $\begin{array}{l}\text { Note }: \\
{ }^{*} \mathrm{p}<0,05 ;{ }^{* * *} \mathrm{p}<0,001 .\end{array}$ & \\
\hline
\end{tabular}

niveau d'adhésion à ces principes est très élevé, plus élevé que l'adhésion au multiculturalisme, ce qui semble valider l'existence de cette tradition spécifique à la France. Contrairement au discours ambiant, il n'y a pas forcément de contradiction entre le multiculturalisme et certains aspects fondamentaux du modèle républicain français (objectif 2). Ces deux idéologies, rappelons-le, divergent pourtant dans leurs approches : l'une défend la reconnaissance des identités et l'autre l'élimination des catégories psychologiquement construites qui pourraient se référer aux diverses appartenances ou origines. Certains auteurs (Ryan, Hunt, Weibe, Peterson, \& Casas, 2007) étudiant l'adhésion à l'idéologie color-blind « aveugle à la couleur » et au multiculturalisme aux États-Unis ont déjà pu observer un tel lien positif entre des conceptions a priori antagonistes. L'idéologie color-blind est ici à rapprocher du principe français de la citoyenneté. En effet, toutes deux reconnaissent et prônent l'égalité des individus sans que la couleur de peau ou encore l'origine ne soit considérée. Ainsi, les analyses de Ryan et ses collègues (2007) montrent que cette idéologie color-blind, bien que conceptuellement distincte, est reliée positivement et non négativement au multiculturalisme (pour les participants Noirs comme pour les Blancs). Toutefois les auteurs n'avancent que peu d'éléments pour expliquer cette corrélation positive. Nos résultats reliés à l'ODS suggèrent en revanche une voie de réponse possible : le principe de citoyenneté en France (comme le color-blind aux USA) partagerait avec le multiculturalisme le fait de relever d'une orientation égalitaire et donc, dans les faits, d'être relié négativement à la dominance sociale (objectif 3 ).

Dans l'ensemble, les résultats obtenus confirment les caractéristiques du « modèle républicain » décrites par Schnapper (2004) incluant l'idée que ce modèle «n'a jamais interdit le «multiculturalisme » dans la vie sociale »(p. 177). En revanche, le principe de laïcité, qui a 
dominé le débat public récemment, apparaît lui comme incompatible «psychologiquement » avec le multiculturalisme et comme un facteur d'ethnocentrisme et non d'ouverture et de tolérance. Ces résultats reflètent la perspective des membres du groupe majoritaire ou dominant. Tel que mentionné précédemment, une troisième et dernière étude a été menée afin d'examiner, à l'exemple de ce que suggère le modèle d'acculturation interactif (Bourhis, Moïse, Perreault, \& Sénécal, 1997), le point de vue de Français d'origine maghrébine en tant que membre d'une minorité culturelle.

\section{ÉTUDE 3}

\section{Méthode \\ Participants}

Avec l'objectif d'identifier des personnes d'origine maghrébine, quarante-deux personnes étaient recrutées dans divers lieux publics de la ville de ClermontFerrand dont 20 femmes (âge moyen $=25,5$ ans ; $E T=9,13$ ) et 22 hommes (âge moyen $=29$ ans $; E T=8,38$ ). Confirmant l'appartenance des participants à un groupe minoritaire en France, $95 \%$ des participants se sont déclarés de religion musulmane, et $5 \%$ sans religion. En moyenne, les participants disaient vivre en France depuis environ 21 ans. Parmi eux, 71,5\% étaient nés en France et 28,5\% étaient nés à l'étranger (Algérie, Maroc, Tunisie ou Turquie). En remerciement de leur participation, ils recevaient un bon d'achat de 5 euros.

\section{Matériel}

Les participants répondaient, dans l'ordre, aux mesures du modèle républicain, du multiculturalisme $(\alpha=0,68)$ et de l'orientation à la dominance sociale $(\alpha=0,75)$ (voir description dans Études 1 et 2). L'échelle de citoyenneté républicaine composée de quatre items possède une fidélité satisfaisante $(\alpha=0,73)$, au contraire de l'échelle de laïcité $(\alpha=0,25)$. Pour cette raison, les résultats reliés à l'échelle de laïcité ne seront pas considérés.

En plus de ces trois mesures, nous avons inclus des mesures de biais intergroupes et de préférences en matière de stratégies d'acculturation (Dambrun \& Guimond, 2001 ; Berry, 2005). Cette dernière comportait 16 items présentés dans un ordre aléatoire. Chaque stratégie était mesurée par 4 items (Intégration « Je pense que les personnes d'origine maghrébine doivent garder leur culture, mais aussi s'adapter à la culture française »; Assimilation « Je pense que les personnes d'origine maghrébine doivent s'adapter aux coutumes françaises et ne pas garder les coutumes maghrébines »; Séparation: « Je pense que les personnes d'origine maghrébine doivent garder leur culture et ne doivent pas s'adapter à la culture 
française »; Marginalisation « Je pense que les personnes d'origine maghrébine ne doivent pas garder leur culture d'origine et ne doivent pas s'adapter à la culture française »). Dans la mesure où les alphas de Cronbach des quatre items étaient satisfaisants ( $\alpha=0,68$ pour l'intégration, $\alpha=0,60$ pour l'assimilation, $\alpha=0,77$ pour la séparation et $\alpha=0,73$ pour la marginalisation), un indicateur pour chaque stratégie a été calculé au travers d'une moyenne de leurs réponses aux quatre items.

Pour mesurer le biais intergroupe, nous présentions aux participants une liste de 18 groupes sociaux (c'est-à-dire " les hommes », « les policiers », etc.) en leur demandant d'indiquer leur attitude générale à l'égard de chacun d'eux à l'aide d'une échelle de 1 (attitude très défavorable) à 7 (attitude très favorable). Parmi cette liste de groupes figurait le groupe des «Maghrébins » et le groupe des « Français ». Une mesure de biais de favoritisme pro-endogroupe était obtenue en comparant l'évaluation de ces deux groupes (voir Dambrun \& Guimond, 2001 pour la validation d'une telle mesure).

\section{Procédure}

Dans la rue, l'expérimentateur (d'apparence européenne ou maghrébine) demandait à des passants s'ils étaient volontaires pour participer à une étude. Lorsque ces derniers acceptaient de participer, l'expérimentateur leur expliquait qu'ils allaient devoir répondre à un questionnaire destiné à mesurer leurs impressions sociales générales. Sur la première page du questionnaire, un certain nombre de consignes figurait pour le bon déroulement de l'étude. Il était indiqué que le participant devait donner ses opinions spontanées et sincères. Il était également mentionné qu'il n'y avait pas de bonnes ou de mauvaises réponses et que l'anonymat des réponses était totalement garanti. Ensuite, l'expérimentateur s'éloignait du participant en lui demandant de venir le chercher lorsqu'il aurait fini. Aucune limite de temps n'était donnée. Après avoir rempli le questionnaire, tous les participants (condition « expérimentateur maghrébin » comme condition « expérimentateur européen ») étaient débriefés et remerciés. Enfin, ils étaient conviés à poser les questions qu'ils souhaitaient et à exprimer leur avis.

\section{Résultats}

\section{Lien entre modèle républicain, multiculturalisme et stratégies d'acculturation}

Dans une série d'analyses corrélationnelles, nous avons souhaité examiner le lien entre la citoyenneté républicaine, le multiculturalisme, l'ODS, et les quatre stratégies d'acculturation ( $c f$. Tableau 6).

Comme attendu, le Tableau 6 montre que les attitudes envers le multiculturalisme sont reliées à des attitudes positives envers la stratégie d'intégration et à des attitudes négatives envers la stratégie d'assimilation. Ceci vient donc renforcer la validité de ces mesures. Cependant, et de manière plus surprenante, le niveau de citoyenneté est négativement 
Tableau 6. Corrélations entre citoyenneté, multiculturalisme, orientation à la dominance sociale et les quatre stratégies d'acculturation

Table 6. Correlations between «citizenship », multiculturalism, social dominance orientation and four acculturation's strategies

\begin{tabular}{lccccccc}
\hline & $\mathbf{1}$ & $\mathbf{2}$ & $\mathbf{3}$ & $\mathbf{4}$ & $\mathbf{5}$ & $\mathbf{6}$ & $\mathbf{7}$ \\
\hline 1. Citoyenneté & - & & & & & \\
\hline 2. Multiculturalisme & $0,57^{* * *}$ & - & & & & \\
\hline 3. ODS & $-0,53^{* * *}$ & $-0,36^{*}$ & - & & & \\
\hline 4. Intégration & $0,51^{* * *}$ & $0,38^{*}$ & $-0,15$ & - & & \\
\hline 5. Assimilation & $-0,50^{* * *}$ & $-0,57^{* * *}$ & $0,38^{*}$ & $-0,44^{* *}$ & - & \\
\hline 6. Séparation & $-0,42^{* *}$ & 0,04 & 0,25 & $-0,16$ & 0,28 & - \\
\hline 7. Marginalisation & $-0,43^{* *}$ & $-0,18$ & $0,38^{*}$ & $-0,27$ & $0,45^{* *}$ & $0,53^{* * *}$ \\
\hline $\begin{array}{l}\text { Note: } \\
\text { (ODS) Orientation à la dominance sociale } ;(N=42)\end{array}$ \\
${ }^{*} p<0,01 ; * * p<0,01 ; * * p<0,001$.
\end{tabular}

corrélé avec l'assimilation, $r(42)=-0,50, p<0,001$, positivement avec l'intégration, $r(42)=0,51, p<0,001$, et avec le multiculturalisme, $r(42)=0,57, p<0,001$. Ces résultats suggèrent que le principe de citoyenneté républicaine n'est pas conforme aux principes d'un modèle assimilationniste. De plus, pour les participants d'origine maghrébine de cette étude, comme pour ceux des études précédentes, la citoyenneté républicaine est reliée négativement à la dominance sociale $(r=-0,53$, $p<0,001)$ tandis que la stratégie d'assimilation est reliée de manière positive à la dominance sociale $(r=0,38, p<0,05)$. Enfin, dans une autre série de tests appariés, on observe que les participants maghrébins se montrent plus favorables à la citoyenneté républicaine $(M=6,25)$ qu'au multiculturalisme $(M=5,48), t(41)=5,25, p<0,001$.

\section{Stratégies d'acculturation et rôle de l'expérimentateur}

Nous avons examiné le choix des stratégies d'acculturation à l'aide d'une analyse de variance. Les résultats montrent que la stratégie 
d'acculturation privilégiée des Maghrébins est l'intégration $(M=6,46)$ alors que l'assimilation $(M=1,99)$, la séparation $(M=2,40)$ et la marginalisation $(M=1,65)$ sont les stratégies les moins choisies, $F(3,123)=189,97, p<0,001$. Des analyses supplémentaires ont été réalisées afin d'explorer le rôle de l'expérimentateur. Les résultats indiquent que le type d'expérimentateur n'a généralement pas d'effet sur les réponses des participants sauf dans le cas de la stratégie d'assimilation. En effet, les participants interrogés par un expérimentateur européen se disent plus assimilationnistes comparés aux participants questionnés par un expérimentateur maghrébin, $t(40)=2,51, p<0,02$. Aucune différence significative n'est observée entre l'expérimentateur européen et maghrébin en ce qui concerne l'intégration, la séparation, et la marginalisation, (respectivement, $t(40)=-0,25, p>0,80, t(40)=1,73, p>0,09$, et $t(40)=2,02, p>0,05)(c f$. Figure 3).

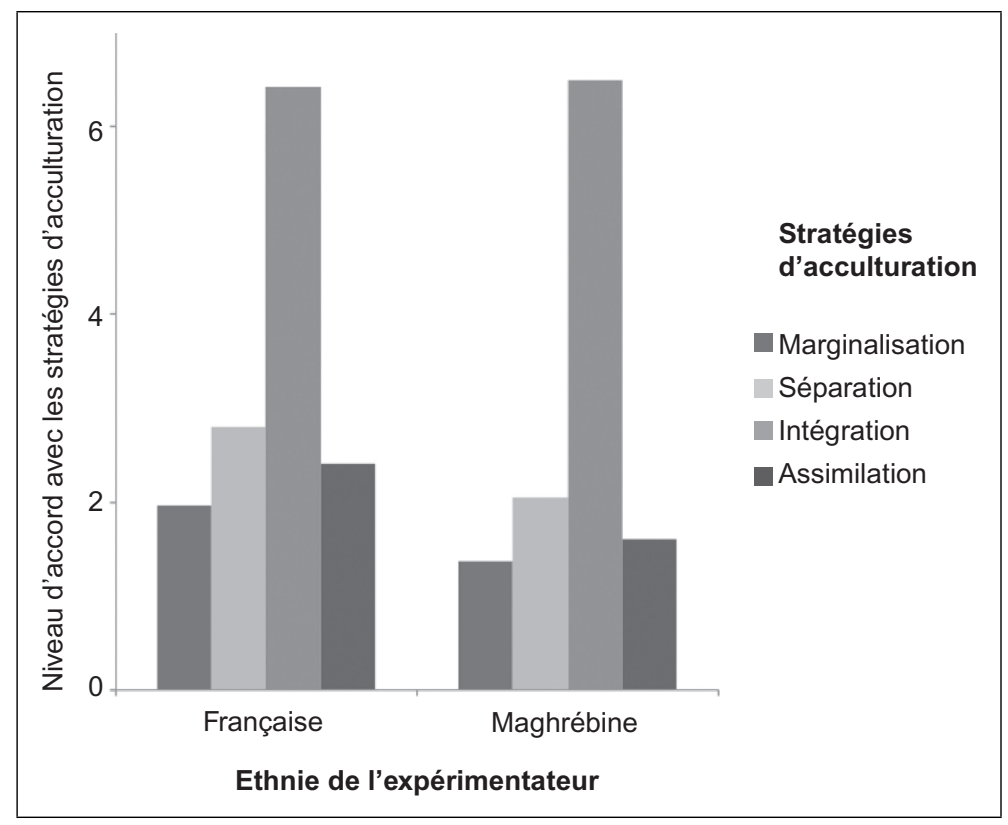

Figure 3. Les stratégies d'acculturation des Maghrébins en fonction de l'ethnie de l'expérimentateur

Figure 3. Acculturation strategies of Maghrebins as a function of the experimenter's ethnicity 


\section{Biais intergroupe et identité de l'expérimentateur}

Les analyses montrent que les participants présentent un biais de favoritisme pro-endogroupe. Plus précisément, les participants expriment une attitude significativement plus favorable à l'égard des Maghrébins $(M=6,31)$ qu'à l'égard des Français $(M=5,51), t(39)=2,55, p<0,02$. Ce biais intergroupe dépend-il de l'ethnie de l'expérimentateur? Afin de répondre à cette question, nous avons effectué une ANOVA à 2 facteurs, ethnie de l'expérimentateur (française vs. maghrébine) et groupe cible évalué (Français vs. Maghrébins) avec mesures répétées sur le dernier facteur. Cette analyse révèle une interaction entre le type d'expérimentateur et le groupe cible évalué, $F(1,37)=4,14, p<0,05$ (voir Figure 4). L'effet principal du groupe cible évalué est également significatif, $F(1,37)=7,30$, $p<0,01$, mais pas celui de l'ethnie de l'expérimentateur, $F(1,37)=1,26, n s$. Des analyses post-hoc montrent que lorsque l'expérimentateur est d'origine maghrébine, les participants évaluent plus positivement les Maghrébins $(M=6,42)$ que les Français $(M=5,00), t(18)=2,28, p<0,02$.

En revanche, quand l'expérimentateur est d'apparence européenne, aucune différence d'évaluation des Maghrébins $(M=6,20)$ et des Français n'est observée $(M=6,00), t(19)=0,61, n s$.

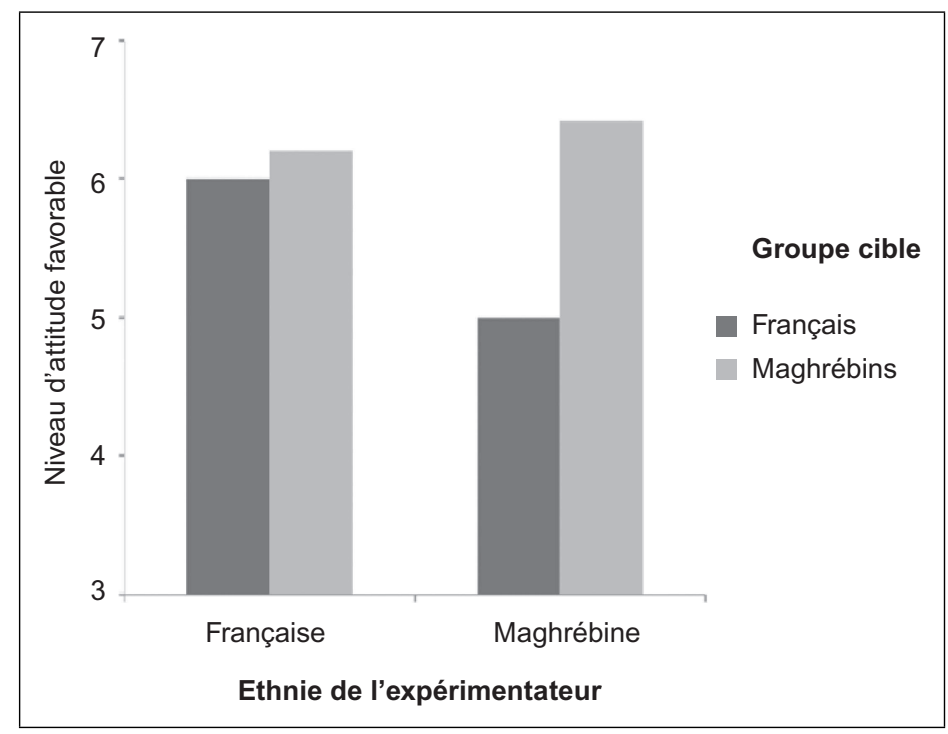

Figure 4. Biais intergroupe en fonction de l'ethnie de l'expérimentateur

Figure 4. Intergroup bias as a function of the experimenter's ethnicity 


\section{Biais intergroupe et citoyenneté républicaine}

La même analyse a été répétée afin d'examiner le rôle de la citoyenneté républicaine. En effet, les Études 1 et 2 ont montré que la citoyenneté est associée à une réduction des préjugés anti-maghrébins. Du point de vue des Maghrébins eux-mêmes, on peut se demander si l'adhésion à ce principe atténue le biais de favoritisme pro-endogroupe. Pour le savoir, nous avons séparé l'échantillon à la médiane pour obtenir un groupe adhérant très fortement et un autre plus faiblement à la citoyenneté. Une ANOVA avec les facteurs citoyenneté (faible vs. élevée) et groupe cible évalué (Français vs. Maghrébins), avec mesures répétées sur le dernier facteur, révèle un effet d'interaction significatif, $F(1,38)=4,61, p<0,04$. La décomposition de cette interaction indique que les participants qui adhèrent faiblement au principe de citoyenneté évaluent les Maghrébins $(M=6,40)$ plus favorablement que les Français, $(M=5,00), t(17)=2,85$, $p<0,01$. En revanche, ce biais intergroupe n'est pas observé chez ceux qui adhèrent fortement au principe de citoyenneté. Ces derniers évaluent les Français $(M=5,90)$ aussi favorablement que les Maghrébins $(M=6,00)$, $t(22)=0,63, n s$. Cet effet de l'adhésion au principe de citoyenneté sur le biais intergroupe demeure significatif même lorsqu'on tient compte de l'identité de l'expérimentateur.

\section{DISCUSSION GÉNÉRALE}

Dans l'ouvrage collectif intitulé Immigration et intégration : L'état des savoirs, Cohen (1999) soulève la question suivante : «l'intégration sociétale est-elle favorisée plutôt par le multiculturalisme ou par une citoyenneté républicaine?» (p. 39). La présente série d'études a proposé une démarche empirique visant à fournir des éléments de réponse à cette question capitale. Postulant qu'un modèle qui favorise l'intégration sociétale devrait avoir des implications positives pour les relations entre groupes, nous avons examiné au travers de quelques études les relations entre l'adhésion à ces deux modèles, le multiculturalisme et le modèle républicain, et le degré de tolérance et d'ouverture manifesté par les individus à l'égard des autres groupes. Le premier constat, qui confirme les recherches antérieures menées principalement hors de France (c'est-à-dire Berry, 2006 ; Verkuyten, 2005), est que le multiculturalisme est relié à des attitudes intergroupes positives. Dans les Études 1 et 2, les participants qui sont favorables au multiculturalisme expriment des niveaux de préjugés 
significativement plus faibles. Afin d'établir de telles relations, nous avons utilisé l'échelle de l'idéologie du multiculturalisme qui a été développée par Berry et Kalin (1995). Cependant, aucun instrument comparable n’a été développé afin de mesurer l'adhésion au modèle républicain français.

Suite à un pré-test, nous avons identifié un certain nombre d'énoncés susceptibles de faire partie d'une telle mesure de l'idéologie républicaine. Dans le pré-test et dans deux études indépendantes, nous observons une structure factorielle de ces items en deux dimensions indépendantes, l'une concernant la citoyenneté et le principe de décatégorisation, l'autre touchant à la laïcité. De plus, alors que l'adhésion au multiculturalisme est relativement élevée (ce qui concorde avec les recherches antérieures), l'adhésion à ces deux dimensions " républicaines » est significativement plus forte, ce qui n'a jamais été mis en évidence auparavant. Ces résultats sont compatibles avec l'idée qu'il existe en France une tradition d'intégration spécifique, qu'on peut appeler le modèle républicain, et qui est distincte du modèle du multiculturalisme.

Ces résultats soulèvent la possibilité que les recherches antérieures, qui n'ont pas tenu compte du modèle républicain, relèvent peut-être d'une image tronquée de la réalité française. Par exemple, Barrette et al. (2004) observent que la stratégie d'intégration (multiculturalisme) est de loin l'orientation d'acculturation préférée par les étudiants français et maghrébins d'universités parisiennes, alors que les stratégies d'assimilation, de séparation et de marginalisation sont rejetées. La cinquième orientation d'acculturation mesurée par Barrette et al. (2004), l'individualisme, est également évaluée favorablement par les participants, à niveau égal de l'intégration. Conceptuellement, cette orientation individualiste est proche de notre mesure de citoyenneté sans toutefois en être identique. Nous observons ainsi une adhésion significativement plus élevée à ce principe de citoyenneté comparativement au multiculturalisme (intégration). De plus, l'individualisme ne possède aucune composante reliée à la laïcité qui reçoit également, dans les Études 1 et 2, une évaluation significativement plus favorable que celle accordée au multiculturalisme. Le fait de considérer de manière explicite les caractéristiques du modèle républicain d'intégration semble donc apporter de nouveaux éléments importants qui ne figuraient pas dans les recherches antérieures. Il serait donc intéressant dans les recherches futures de confronter directement les principes du modèle républicain à cette orientation individualiste.

Les analyses examinant les relations entre l'adhésion à la citoyenneté, à la laïcité et les autres variables pertinentes renforcent l'idée que ces 
facteurs ne se réduisent pas à une question d'individualisme. Confirmant l'indépendance de ces deux dimensions, nous observons dans les Études 1 et 2 que la citoyenneté et la laïcité sont reliées de manière diamétralement opposée au multiculturalisme et aux préjugés. La citoyenneté est reliée de manière positive au multiculturalisme et de manière négative aux préjugés. Ces résultats confirment, en milieu naturel, l'hypothèse du modèle de la personnalisation de Brewer et Miller (1984). Conformément à ce modèle, les individus qui, en France, refusent la catégorisation en termes ethniques, culturels ou religieux au profit de l'unique reconnaissance de citoyens égaux devant la loi (idéologie au coeur de la Révolution française), manifestent significativement moins de préjugés comparativement à celles et ceux qui n'adhèrent pas à ce principe. Cependant, l'adhésion au principe de citoyenneté est aussi reliée à des attitudes positives envers le multiculturalisme. Ce résultat est surprenant pour au moins deux raisons. D'abord, étant donné la forte opposition habituellement établie dans le discours public en France entre multiculturalisme et modèle républicain, on ne s'attendrait pas à de tels liens positifs. Ensuite, au niveau théorique, le principe de décatégorisation du modèle de Brewer et Miller (1984) apparait comme antagoniste avec une approche du type multiculturalisme, qui se propose d'accentuer la saillance de la catégorisation sociale. Par conséquent, on s'attendrait plutôt à des relations négatives entre le principe de citoyenneté et les attitudes envers le multiculturalisme. Malgré ces apparentes contradictions, divers éléments viennent appuyer la validité de nos résultats.

D'abord, nous observons ces relations positives entre citoyenneté et multiculturalisme auprès de trois échantillons distincts et indépendants, incluant un échantillon de Français d'origine maghrébine. Ensuite, ces résultats sont compatibles avec les recherches existantes. Barrette et al. (2004) observent également que l'individualisme (au sens de ne pas tenir compte des appartenances groupales) est relié positivement à l'orientation d'intégration qui implique pourtant d'accorder de l'importance aux appartenances culturelles. Ryan et ses collègues (2007) observent aussi dans deux études auprès de Blancs et de Noirs aux États-Unis, que l'adhésion à l'idéologie du color-blind est corrélée positivement à l'idéologie du multiculturalisme. Or le color-blind implique aussi, comme le principe de citoyenneté républicaine, la notion de décatégorisation. Il nous parait donc difficile de considérer ces résultats comme de simples artefacts. Comment alors expliquer que plus on adhère à la citoyenneté républicaine, plus on est favorable au multiculturalisme ? Nos résultats en rapport à l'orientation de dominance sociale suggèrent une voie possible de réponse à cette interrogation. 
En effet, notre mesure de citoyenneté est reliée à l'échelle ODS mais uniquement à la composante de cette échelle traitant de l'égalité sociale, pas à la composante traitant de la domination. Ainsi, plus les individus adhèrent au principe de citoyenneté, plus ils sont favorables à l'égalité sociale. Or, le multiculturalisme est aussi une orientation égalitaire qui est reliée négativement à la dominance sociale. En somme, la citoyenneté républicaine et le multiculturalisme partagent en commun le fait d'être deux orientations égalitaires, des idéologies qui visent à atténuer les inégalités et la hiérarchie sociale (Guimond, Kamiejski, \& Kang, 2008), ce qui permet d'expliquer les relations positives existant entre elles.

Autre résultat révélateur, les attitudes positives envers le principe de laïcité ne sont reliées ni au principe de citoyenneté, ni à la dominance sociale. Ceci confère un statut distinct au principe de laïcité dont l'évaluation est sans doute davantage tributaire des débats médiatiques entourant la loi interdisant le port de symboles religieux dans les écoles publiques en France, une atmosphère qui exacerbe tensions et sentiments d'hostilité. D'ailleurs, historiquement, la laïcité est une valeur qui a surtout été portée et défendue par la gauche française. Or, l'enquête de Barthélemy et Michelat (2007) suggère que plus récemment, une nouvelle appropriation politique de ce principe viserait à l'utiliser comme moyen de dissimulation de certains préjugés envers les Maghrébins et les Musulmans de France. Nos résultats rejoignent en partie cette analyse en montrant très clairement que les Français les plus favorables à la laïcité manifestent plus, et non moins, de préjugés anti-maghrébins. De plus, l'incompatibilité attendue entre le modèle français et le modèle du multiculturalisme se retrouve bel et bien lorsqu'on considère ce principe de laïcité. En effet, dans les Études 1 et 2, les attitudes positives envers la laïcité sont reliées à des attitudes négatives envers le multiculturalisme.

L'Étude 3 permet de compléter cette vue d'ensemble en considérant la réaction des membres du groupe culturel minoritaire le plus important en France. Malheureusement, nous observons ici que l'échelle de laïcité n'est pas un instrument approprié auprès de cette population. D'autres travaux seront nécessaires afin de développer une mesure des attitudes reliées à la laïcité qui soit valable pour cette population. De manière plus générale, il est évident que la laïcité en France recouvre une réalité complexe et multidimensionnelle à l'égard de laquelle la présente recherche ne rend pas justice (voir Barthélemy \& Michelat, 2007). En ce sens, l'échelle de laïcité présentée ici doit être considérée comme une mesure de certaines attitudes reliées à certains aspects de la laïcité. D'autres recherches sont nécessaires et les premiers résultats présentés ici ne peuvent servir qu'à attirer l'attention 
des chercheurs sur l'importance de cette question de la laïcité en France. En revanche, l'échelle de citoyenneté apparaît comme un instrument fiable, y compris auprès des personnes d'origine maghrébine dont elle prédit les orientations d'acculturation.

Plus précisément, l'Étude 3 confirme auprès de personnes originaires du Maghreb et de religion musulmane que l'adhésion au principe de citoyenneté républicaine est plus forte que celle à l'égard du multiculturalisme et qu'il n'y a pas d'incompatibilité psychologique entre ces deux modèles. De plus, la citoyenneté est reliée négativement à l'ODS, répliquant les résultats des Études 1 et 2. Enfin, par rapport aux stratégies d'acculturation du modèle de Berry (2005), les résultats montrent que le principe de citoyenneté républicaine est relié positivement à la stratégie d'intégration mais négativement à l'assimilation, la séparation ou la marginalisation. Ces résultats sont importants pour plusieurs raisons.

Considérant que le modèle français est souvent présenté comme étant essentiellement un modèle assimilationniste (Barrette et al., 2004 ; Sabatier \& Berry, 1999), ces résultats montrent les limites d'une telle conception. De toute évidence, il existe une composante d'assimilation dans la tradition française et on la retrouve dans la relation négative entre le principe de laïcité et les attitudes envers le multiculturalisme. La loi sur les symboles religieux à l'école a été défendue au nom de la laïcité mais elle porte une visée clairement assimilationniste qui revendique le conformisme aux valeurs de la majorité. Cependant, avec la citoyenneté républicaine, nos résultats montrent qu'il existe aussi une autre composante dans le modèle français qui n'est pas forcément assimilationniste. Ainsi, non seulement l'adhésion au principe de citoyenneté est reliée chez les membres du groupe majoritaire en France à des attitudes positives envers le multiculturalisme, mais les personnes d'origine maghrébine qui adhèrent davantage à ce principe souhaitent aussi moins s'assimiler et plus s'intégrer au sens du modèle de Berry (2005). Ces résultats ne sont donc pas compatibles avec l'idée que la politique française est exclusivement assimilationniste. Ils mettent en évidence une nouvelle dimension du modèle français qui semble des plus favorables à l'intégration sociétale au sens de Cohen (1999).

Les résultats du biais intergroupe des Maghrébins dans l'évaluation des Français et des Maghrébins viennent également plaider pour l'efficacité du modèle républicain, ou du moins de certains principes qui lui sont reliés. En effet, l'adhésion à la citoyenneté républicaine réduit le biais intergroupe des Maghrébins et se traduit par une évaluation positive et égalitaire des deux groupes. Dans une perspective assimilationniste, les Maghrébins devraient 
dévaloriser leur groupe et évaluer le groupe majoritaire plus favorablement. Or, nos résultats montrent qu'ils manifestent une évaluation positive de leur groupe. Le rôle de l'appartenance ethnique de l'expérimentateur apparait ici comme important. Avec un expérimentateur membre du groupe majoritaire, les Maghrébins évaluent les deux groupes de manière égalitaire, alors qu'avec un expérimentateur maghrébin ils ont plus tendance à manifester un biais en faveur de leur propre groupe. Ceci suggère l'existence d'une saine estime de soi collective (Wright \& Taylor, 1995). De la même manière, les participants maghrébins se disent davantage orientés vers l'assimilation avec un expérimentateur majoritaire. Ceci montre bien qu'il existe des normes dont il faut tenir compte dans l'étude des stratégies d'acculturation (voir Taillandier \& Maisonneuve, 2005). Plus généralement, l'Étude 3 montre qu'il est primordial que les recherches futures contrôlent l'appartenance culturelle de l'expérimentateur. De nombreuses recherches qui observent un biais favorable à l'exogroupe rapportent des résultats qui sont probablement biaisés du fait que ce facteur n'ait pas été contrôlé (voir Guimond \& Dambrun, 2003).

En conclusion, dans cette série de recherches, nous avons identifié deux dimensions indépendantes et orthogonales structurant l'idéologie républicaine, la citoyenneté et la laïcité. Les résultats suggèrent que ces deux composantes sont importantes pour comprendre les attitudes et les perceptions intergroupes en France. Dans la mesure où, comme le soutient Weil (2008) dans les propos cités en exergue, l'égalité est une valeur centrale de la nation française, c'est dans le principe de citoyenneté qu'on la retrouve aujourd'hui et non dans celui de laïcité.

L'incidence de ces principes en matière de préjugés laisse penser qu'ils pourraient intervenir à plus grande échelle dans l'évolution même des préjugés au sein de l'opinion publique en France. Ainsi, la cristallisation régulière du débat public autour de la question de la laïcité, à l'initiative de gouvernements de droite, plutôt que de la citoyenneté républicaine, permettrait peut-être de comprendre les évolutions de ces dernières années en matière d'ethnocentrisme (augmentation pour les Français les moins ethnocentriques, Mayer, Michelat \& Tiberj, 2010). Toutefois, d'autres recherches sont nécessaires afin notamment d'évaluer la généralité des résultats présentés ici. En effet, nos échantillons étaient pour l'essentiel composés de diplômés du supérieur, nés après 1977. On peut se demander si de nouvelles cohortes de naissance, antérieures aux années 1980 présenteraient les mêmes réactions en matière de préjugés (l'intolérance augmentant avec l'âge et diminuant avec le niveau d'étude) et selon la nature de ceux-ci (le type de racisme par exemple ou encore 
d'aversion). Dans cette optique, une enquête auprès d'un échantillon représentatif de la population française semblable à celle réalisée par Barthélemy et Michelat (2007) sur la laïcité mais incluant une mesure de la citoyenneté serait particulièrement informative. Enfin, alors qu'il semble pertinent de considérer l'intégration républicaine comme cadre interprétatif à la légitimation des préjugés en France, il paraîtrait également opportun de considérer son impact direct sur les préjugés notamment au travers de futures études visant à induire expérimentalement ce modèle dans la suite des travaux de Wolsko, Park, Judd, et Wittenbrink (2000).

Reçu le 1er juin 2010.

Révision acceptée le 15 février 2011.

\section{BIBLIOGRAPHIE}

Allport, G. (1954). The nature of prejudice. Reading, MA: Addison-Wesley.

Amadieu, J. -F. (2004). Enquête « Testing 》 sur CV. Paris : l'Observatoire des discriminations.

Amadieu, J. -F. (2006). Baromètre Adia. Paris : l'Observatoire des discriminations.

Barrette, G., Bourhis, R. Y., Personnaz, M., \& Personnaz, B. (2004). Acculturation orientations of French and North African undergraduates in Paris. International Journal of Intercultural Relations, 28, 415-438.

Barthélemy, M., \& Michelat, G. (2007). Les représentations de la laïcité chez les Français. In P.Weil, (Eds.), Politiques de la laïcité au XX siècle (pp. 603-629). Paris : PUF.

Barthélémy, M. \& Michelat, G. (2007). Dimension de la laïcité dans la France d'aujourd'hui. Revue française de science politique, 57, 649-698

Baubérot, J. (2010). Histoire de la laïcité en France. Paris : PUF.
Beauchemin, C., Hamel, C., Lesné, M., Simon, P., \& l'équipe de l'enquête $\mathrm{TeO}$ (2010). Les discriminations : une question de minorités visibles. Population et sociétés, 466.

Bénichou, M. (2006). Le multiculturalisme. Bréal.

Berry, J. W. (1994). De la psychologie culturelle et ethnique : une perspective écologique. Cahiers Internationaux de Psychologie Sociale, 24, 9-32.

Berry, J. W. (2005). Acculturation: Living successfully in two cultures. International Journal of Intercultural Relations, 29, 697-712.

Berry, J. W. (2006). Mutual attitudes among immigrants and ethnocultural groups in Canada. International Journal of Intercultural Relations, 30, 719-734.

Berry, J. W. \& Kalin, R. (1995). Multicultural and ethnic attitudes in Canada. Canadian Journal of Behavioural Science, 27, 310-320. 
Berry, J. W., Kalin, R., \& Taylor, D. M. (1977). Attitude à l'égard du multiculturalisme et des groupes ethniques au Canada. Ottawa : Approvisionnements et Services Canada.

Berry, J. W., Kim, U., Power, S., Young, M., \& Bujaki, M. (1989). Acculturation attitudes in plural society. Applied Psychology: An International Review, 38, 185-206.

Bertossi, C. (2006). How does the French Republic deal with Ethno-cultural and Religious Diversity? Londres: Runnymede Trust.

Bouhris, R. Y., Moïse, L. C., Perreault, S., \& Sénécal, S. (1997). Towards an interactive acculturation model: a social psychological approach. International Journal of Psychology, 32, 369-386.

Brauer, M., Wasel, W., \& Niedenthal, P. (2000). Implicit and explicit components of prejudice. Review of General Psychology, 4, 79-101.

Brewer, M. B., \& Miller, M. (1984). Beyond the contact hypothesis: theoretical perspectives and desegregations. In M.Miller, \& M. B.Brewer, (Eds), Groups in contact: The psychology of desegregations. New-York: Academic press.

Brouard, S., \& Tiberj, V. (2005). Français comme les autres? Paris: Presses de Sciences Po.

Bruner, J. S. (1957). On perceptual readiness. Psychological Review, 64, 123-152.

Cohen, J. (1999). Intégration : Théories, politiques et logiques d'État. In P.Dewitte, (Ed.), Immigration et intégration: L'état des savoirs (pp. 32-42). Paris : La découverte.

Dambrun, M., \& Guimond, S. (2001). La théorie de la privation relative et l'hostilité envers les Nord-Africains. Revue Internationale de Psychologie Sociale/International Review of Social Psychology, 14, 57-89.

De Rudder, V., Poiret, C., \& Vourc'h, F. (2000). L'Inégalité raciste : L'Universalité républicaine à l'épreuve. Paris : PUF.

Devine, P. G. (1989). Stereotypes and prejudice: Their automatic and controlled components. Journal of Personality and Social Psychology, 56, 5-18.

Duarte, S., Dambrun, M., \& Guimond, S. (2004). La théorie de la dominance sociale et « les mythes légitimisateurs » : Validation d'une version française de l'échelle d'orientation de dominance sociale. Revue Internationale de Psychologie Sociale /International Review of Social Psychology, 17, 97-126.

Felouzis, G. (2006). Ethnicité et ségrégation dans les écoles françaises. In J. Polard, A.-G. Gagnon, \& B.Gagnon, (Eds), Diversité et identités au Québec et dans les régions d'Europe (pp. 305-318). Québec : Presses de l'Université Laval.

Furedi, F. (2005). Courrier International, $785,48-49$

Guimond, S. (1999). Le multiculturalisme comme politique de gestion de la diversité culturelle. In W. Doise, , N. Dubois, \& J. L. Beauvois, (Eds), La psychologie sociale (Tome 4) : La construction sociale de la personne (pp. 173-181). Grenoble : Presses Universitaires de Grenoble.

Guimond, S. (2004). Lutter contre le racisme et le sexisme en milieu scolaire. In M.-C. Toczek, \& D. Martinot, . Le défi éducatif. (pp. 169-347). Paris : Armand Colin.

Guimond, S. (2010). Psychologie sociale: Perspective multiculturelle. Bruxelles : Mardaga.

Guimond, S., \& Dambrun, M. (2003). La construction sociale et scolaire du stigmate. In J.-C. Croizet, \& J.-P. Leyens, (Eds.), Mauvaises réputations (pp.177-213). Paris : Armand Colin.

Guimond, S., Kamiejski, R., \& Kang, P. (2008). Psychologie de la dominance sociale : hiérarchie sociale et relations entre groupes. In R. V. Joule, \& P. Huguet, (Eds.), Bilans et perspectives en psychologie sociale (II). PUG.

Henry, P. J. (2008). Student sampling as a theoretical problem. Psychological Inquiry, 19, 114-125. 
Hewstone, M., \& Brown, R. (1986). Content is not enough: An intergroup perspective on the contact hypothesis. In M.Hewstone, \& R.Brown, (Eds.), Contact and Conflict in Intergroup Encounters (pp. 1-44). Oxford, UK: Basil Blackwell.

Jost, J. T., \& Thompson, E. P. (2000). Group-based dominance and opposition to equality as independent predictors of self-esteem, ethnocentrism, and social policy attitudes among African Americans and European Americans. Journal of Experimental Social Psychology, 36, 209-232.

Lambert, W. E., Moghaddam, F. M., Sorin, J., \& Sorin, S. (1990). Assimilation vs. multiculturalism: Views from a community in France. Sociological Forum, 5, 387-411.

Maisonneuve, C., \& Testé, B. (2007). Acculturation preferences of a host community: The effects of immigrant acculturation strategies on evaluations and impression formation. International Journal of Intercultural Relations, 31, 669-688.

Macrae, C. N., Bodenhausen, G. V., Milne, A. B., \& Jetten, J. (1994). Out of mind but back in sight: Stereotypes on the rebound. Journal of Personality and Social Psychology. 67, 808-817.

Mayer, N., \& Michelat, G. (2007). Les transformations du rapport à l'autre: le rôle des identités politiques et religieuses. In Commission Nationale Consultative des Droits de l'Homme, La lutte contre le racisme, l'antisémitisme et la xénophobie, année 2006. Paris, La Documentation française.

Mayer, N., Michelat, G., \& Tiberj, V. (2010). Le racisme à l'heure de la crise. In Commission nationale consultative des droits de l'homme, La lutte contre le racisme, l'antisémitisme et la xénophobie, année 2009. La Documentation Française. 102-123.

Mayer, N., Michelat, G., \& Tiberj, V. (2008). Etranger, immigré, musulman: les representations de "l'autre" dans la société française0, In Commission nationale Consultative des Droits de l'Homme, La lutte contre le racisme, l'antisémitisme et la xénophobie, année 2007. Paris: La Documentation Française.

Miller, N. (2002). Personalization and the promise of contact theory. The Journal of social issues, 58(2), 387-410.

Observatoire Européen des Phénomènes Racistes et Xénophobes (EUMC), (2006). Perceptions de la discrimination et de l'islamophobie. Point de vue de membres des communautés musulmanes dans l'union européenne. (http://Eumc.europa.eu).

Paluck, E. L., \& Green, D. P. (2009). Prejudice reduction: What works? A review and assessment of research and practice. Annual Review of Psychology, 60, 339-369.

Park, B., \& Judd, C. M. (2005). Rethinking the Link Between Categorization and Prejudice Within the Social Cognition Perspective. Personality and Social Psychology Review, 9, 108-130.

Pettigrew, T. F., Jackson, J. S., Ben Bricka, J., Lemaine, G., Meertens, R. W., Wagner, U., \& Zick, A. (1998). Outgroup prejudice in Western Europe. European Review of Social Psychology, 8, 241-273.

Reynier (2000). Différents modèles d'intégration nationale. www.reynier.com/ ANTHRO/Interethnique/Modele.html

Richeson, J. A., \& Nussbaum, R. J. (2004). The impact of multiculturalism versus color-blindness on racial bias. Journal of Experimental Social Psychology, 40, 417-423 Ryan, C. S., Hunt, J. S., Weibe, J. A., Peterson, C. R., \& Casas, J. F. (2007). Multicultural and colorblind ideology, stereotypes, and ethnocentrism among Black and White Americans. Group Processes \& Intergroup Relations, 10, 617-637.

Sabatier, C., \& Berry, J. W. (1999). Immigration et acculturation. In R.Y.Bourhis, \& J.-P.Leyens, (Eds), Stéréotypes, discrimination et relations intergroupes. Liège : Pierre Mardaga.

Sala Pala, V., \& Simon, P. (2007). Public and political debates on multicultural crises in France. Report on national and European level cases: EMILIE. 
Schadron, G., \& Morchain, P. (2008). Déterminabilité et contrôle des influences non conscientes. In R. V.Joule, \& P.Huguet, (Eds.), Bilan et Perspectives en Psychologie Sociale, 2, 139-162. Grenoble: Presses Universitaires de Grenoble.

Sherif, M., \& Sherif, C. W. (1953). Groups in Harmony and Tension. New York: Harper.

Schnapper, D. (2000). L'universel républicain revisité. VEI Enjeux, 121, juin.

Schnapper, D. (2004). La République face aux communautarismes. Études 2004/2, 400, 177-188.

Schnapper, D. (2007). Qu'est-ce que l'intégration ? Paris : Gallimard.

Sidanius, J., \& Pratto, F. (1999). Social Dominance: An intergroup theory of social hierarchy and oppression. New York: Cambridge University Press.

Streiff-Fénart, J. (2002). Modèle républicain et discriminations ethniques : Un dilemme français. Faire Savoirs, 1, 69-76.

Taillandier, A., \& Maisonneuve, C. (2005). Mise en évidence d'une norme de non racisme dans la mesure des orientations d'acculturation de la communauté d'accueil. Cahiers Internationaux de Psychologie Sociale, 67-68, 33-53.

Tajfel, H. (1970). Experiments in intergroup discrimination. Scientific American, 223, 96-102.

Tajfel, H., \& Turner, J. C. (1979). Integrative theory of intergroup conflict. In W. G. Austin, \& S.Worchel, (Eds), The social psychology of intergroup relations. Belmont, CA: Wadsworth.
Verkuyten, M. (2005). Ethnic group identification and group evaluation among minority and majority groups: testing the multiculturalism hypothesis. Journal of Personality and Social Psychology, 88, 121-138. Verkuyten, M. (2006). Multicultural recognition and ethnic minority rights: A social identity perspective. European Review of Social Psychology, 17, 148-184.

Verkuyten, M., \& Kwa, G. A. (1996). Ethnic self-identication, ethnic involvement, and group differentiation among Chinese youth in the Netherlands. Journal of Social Psychology, 136, 35-48.

Weil, P. (2007). Politique de la laïcité au XX siècle. Paris : PUF.

Weil, P. (2008). Liberté, Égalité, Discriminations. L'《 identité nationale 》 au regard de l'histoire. Paris : Grasset.

Wolsko, C., Park, B., Judd, C. M., \& Wittenbrink, B. (2000). Framing interethnic ideology: Effects of multicultural and colorblind perspectives on judgments of groups and individuals. Journal of Personality and Social Psychology, 78, 635-654

Wolsko, C., Park, B., \& Judd, C. M. (2006). Considering the tower of Babel: Correlates of assimilation and multiculturalism among ethnic minority and majority groups in the United States. Social Justice Research, 19, 277-306

Wright, S. C., \& Taylor, D. M. (1995). Identity and the language of the classroom: Investigating the impact of heritage versus second language instruction on personal and collective self-esteem. Journal of Educational Psychology, 87, 241-252. 


\section{APPENDICE}

Tableau 3. Facteurs de l'échelle d'adhésion au modèle Républicain après rotation varimax et poids factoriel de chacun des items

Table 3. Factors of Republican model's scale membership after varimax rotation and factor scores of each item

\begin{tabular}{lcc}
\hline $\begin{array}{l}\text { Énoncé de l'item } \\
\text { Cacteur 1 : } \\
\text { Citoyenneté }\end{array}$ & $\begin{array}{c}\text { Facteur 2 : } \\
\text { Laïcité }\end{array}$ \\
\hline $\begin{array}{l}\text { Je ne veux pas qu'on définisse les gens en } \\
\text { France en fonction de leurs origines, de } \\
\text { leur religion. }\end{array}$ & 0,79 & $-0,06$ \\
\hline $\begin{array}{l}\text { La société française est composée de } \\
\text { citoyens avant toute chose et non pas de } \\
\text { communautés. }\end{array}$ & 0,78 & 0,03 \\
\hline $\begin{array}{l}\text { Un Français doit être pris en considération } \\
\text { en tant que tel et pas en tant que membre } \\
\text { d'une communauté particulière (ethnique, } \\
\text { religieuse, sexuelle ...) comme c'est le cas } \\
\text { dans d'autres pays. }\end{array}$ & 0,78 & 0,04 \\
\hline $\begin{array}{l}\text { Pour l'unité du pays, les individus doivent } \\
\text { être considérés comme citoyen avant de } \\
\text { l'être comme black, blanc, beur, femme, } \\
\text { homme, gay ou hétéro. }\end{array}$ & 0,73 & $-0,1$ \\
\hline $\begin{array}{l}\text { Il serait normal qu'en France, l'État finance } \\
\text { la construction de mosquées, de } \\
\text { synagogues et de temples. (inversé) }\end{array}$ & $-0,23$ & \\
\hline $\begin{array}{l}\text { Je trouve qu'en France on va trop loin } \\
\text { lorsque l'on interdit, par principe de } \\
\text { laïcité, le financement public pour la } \\
\text { construction d'édifices religieux. (inversé) }\end{array}$ & & 0,64 \\
\hline $\begin{array}{l}\text { Cela ne me dérange pas que sur la photo de } \\
\text { la carte d'identité française, les catholiques } \\
\text { ou les musulmanes conservent un signe } \\
\text { marquant leur confession (le voile par ex.). } \\
\text { (inversé) }\end{array}$ & $-0,09$ & \\
\hline & & 0,69 \\
& & \\
\hline
\end{tabular}


Tableau 3. (Suite)

\begin{tabular}{lcc}
\hline Énoncé de l'item & $\begin{array}{c}\text { Facteur 1 : } \\
\text { Citoyenneté }\end{array}$ & $\begin{array}{c}\text { Facteur 2 : } \\
\text { Laïcité }\end{array}$ \\
\hline $\begin{array}{l}\text { Autant que possible, les pratiques } \\
\text { religieuses devraient être à caractère privé } \\
\text { et non publique. }\end{array}$ & $-0,08$ & 0,64 \\
\hline $\begin{array}{l}\text { Il me semble tout à fait normal que dans } \\
\text { les écoles publiques en France les signes } \\
\text { religieux visibles soient proscrits } \\
\text { (interdits). }\end{array}$ & 0,22 & 0,63 \\
\hline $\begin{array}{l}\text { Je suis favorable à la séparation du } \\
\text { religieux et de l'État en France comme } \\
\text { dans les autres pays. }\end{array}$ & 0,21 & 0,58 \\
\hline \begin{tabular}{l} 
Pourcentage de variance expliquée \\
\hline
\end{tabular} & 27 & 24 \\
\hline
\end{tabular}




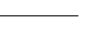

- 